\title{
Diversity and evolution of Tat LTR retrotransposon structures in non-flowering plants
}

\author{
Mikhail Biryukov \\ Interinstitutional laboratory of molecular \\ paleogenetics and paleogenomics \\ ICG SB RAS \\ Novosibirsk, Russia \\ birykov@bionet.nsc.ru
}

\author{
Kirill Ustyantsev \\ The sector of molecular-genetic \\ mechanisms of regeneration \\ ICG SB RAS \\ Novosibirsk, Russia \\ ORCID 0000-0003-4346-3868

\section{Methods and Algorithms}

Abstract - Retrotransposons (RTs) can be characterized as
assemblies of protein domains each of which can have an independent evolutionary fate. This led to a modular view on RTs evolution. An illustrative example is independent acquisition of additional ribonuclease $\mathrm{H}(\mathrm{aRNH})$ domains by distant evolutionary lineages of long terminal repeat (LTR)-RTs in genomes of plants and parasitic protists oomycetes and vertebrate retroviruses. In all the cases structures of the elements converged to a single 'retroviral' variant when the aRNH domain is fixed right after the original one [1, 2]. Only LTR RTs of the Tat clade in plants exhibit structural diversity with various positions of aRNH in relation to the other domains. However, previous study of Tat LTR-RTs diversity was mostly limited by flowering plants [1]. Currently, numerous genomes of non-flowering plants assemblies are available for the analysis, opening a perspective to study general tendencies in Tat LTRRTs evolution in distant plant taxa. We analyzed 78 genome assemblies of gymnosperms, ferns, clubmosses, mosses, and green algae. Our phylogenetic analysis revealed another example of convergent evolution to the 'retroviral' structure in plant LTR-RTs outside Tat, supporting its selective advantage.

Keywords - LTR retrotransposons, convergent evolution, ribonuclease $\mathrm{H}, \mathrm{RNH}, \mathrm{Tat}$

\section{Introduction}

Retrotransposons can be characterized as assemblies of protein domains each of which can have an independent evolutionary fate. This led to a modular view on RTs evolution. An illustrative example is independent acquisition of additional ribonuclease $\mathrm{H}$ (aRNH) domains by distant evolutionary lineages of long terminal repeat (LTR)-RTs in genomes of plants and parasitic protists oomycetes and vertebrate retroviruses. In all the cases structures of the elements converged to a single 'retroviral' variant when the aRNH domain is fixed right after the original one [1], [2]. Only LTR RTs of the Tat clade in plants exhibit structural diversity with various positions of aRNH in relation to the other domains. However, previous study of Tat LTR-RTs diversity was mostly limited by flowering plants [1]. Currently, numerous genomes of non-flowering plants assemblies are available for the analysis, opening a perspective to study general tendencies in Tat LTR-RTs evolution in distant plant taxa.
We implemented a standalone pipeline written in Python which uses NCBI RPS-BLAST and CDD to mine LTR-RTs sequences with aRNH in genome assemblies, automatically annotates domain structure of the identified elements, clusters them and returns non-redundant set of best-scored representative sequences for phylogenetic analysis. The phylogeny was evaluated using IQ-TREE.

\section{Results}

We analyzed 78 genome assemblies of gymnosperms, ferns, clubmosses, mosses, and green algae. Four LTR-RTs structures differing in aRNH position were identified, one of which with aRNH before GAG found in clubmosses is observed for the first time. LTR-RTs from ferns and some clubmosses formed a distinct cluster outside Tat, where the majority of elements have the 'retroviral' structure. The phylogenetic analysis demonstrated that acquisition of aRNH by ferns and clubmosses appears to be independent from the Tat clusters. Among coniferous gymnosperms all previously reported structures were confirmed, while in ancient Ginkgo biloba and Gnetum montanum LTR-RTs with the 'retroviral' and typical gymnosperm variant were found. Phylogenetic analysis of aRNH supports origin of the 'retroviral' structure in Tat before the split between G. biloba, G. montanum and flowering plants.

\section{Conclusion}

Our phylogenetic analysis revealed another example of convergent evolution to the 'retroviral' structure in plant LTRRTs outside Tat, supporting its selective advantage.

\section{Acknowledgment}

Supported by the Russian State Budget Project № 02592019-0010, and № 0324-2019-0040-C-01.

\section{References}

[1] K. Ustyantsev, O. Novikova, A. Blinov, and G. Smyshlyaev, "Convergent Evolution of Ribonuclease H in LTR Retrotransposons and Retroviruses," Mol. Biol. Evol., vol. 32, no. 5, p. 1197, May 2015.

[2] K. Ustyantsev, A. Blinov, and G. Smyshlyaev, "Convergence of retrotransposons in oomycetes and plants," Mob. DNA, vol. 8, no. 1, p. 4, Dec. 2017 